\title{
Deriving Driver Behavioral Pattern Analysis and Performance Using Neural Network Approaches
}

\author{
Meenakshi Malik ${ }^{1}$, Rainu Nandal ${ }^{1, *}$, Surjeet Dalal ${ }^{2}$, Vivek Jalglan ${ }^{3}$ and Dac-Nhuong Le ${ }^{4,5}$ \\ ${ }^{1}$ Department of Computer Science and Engineering, U.I.E.T, Maharshi Dayanand University, Rohtak, 124001, India \\ ${ }^{2}$ Department of Computer Science and Engineering, SRM University, Delhi-NCR, Sonipat, 131029, India \\ ${ }^{3}$ DPG Institute of Technology and Management, Gurugram, 122001, India \\ ${ }^{4}$ School of Computer Science, Duy Tan University, Danang, 550000, Vietnam \\ ${ }^{5}$ Institute of Research and Development, Duy Tan University, Danang, 550000, Vietnam \\ *Corresponding Author: Rainu Nandal. Email: rainu_nandal@yahoo.com \\ Received: 17 May 2021; Accepted: 03 July 2021
}

\begin{abstract}
It has been observed that driver behavior has a direct and considerable impact upon factors like fuel consumption, environmentally harmful emissions, and public safety, making it a key consideration of further research in order to monitor and control such related hazards. This has fueled our decision to conduct a study in order to arrive at an efficient way of analyzing the various parameters of driver behavior and find ways and means of positively impacting such behavior. It has been ascertained that such behavioral patterns can significantly impact the analysis of traffic-related conditions and outcomes. In such cases, the specific vehicular behavior can be detected and related data mined in order to analyze the spatial or temporal patterns of movement patterns as well as to position/track the prominent trends. This analysis seeks to determine the efficacy of such an exercise and whether the various parameters employed can help efficiently determine the various criteria for defining the driver's style. To that end, the analysis of a driver's behavioral pattern and performance utilizes a computer modeled application for generating a set of classifications based on the autonomous driving data as well as indicators that are characteristic of driver aggression. In order to draw such insights from the driver's behavior, the application is modeled using various categories of data, for instance, the steering wheel's angle, braking conditions, acceleration conditions, the vehicle speed, etc. Unlike the previously developed mechanisms for analyzing the system-based driver behavioral patterns, which were not very efficacious, this endeavor assimilates the contemporary breakthroughs in real-world scenario analysis approaches and driver behavior classification methods. Based on the system capabilities and desired outcomes, distinct strategies can be employed in order to detect the target driver's behavior. In this specific case, neural network algorithms were utilized in order to conduct an intensive study to determine and analyze the prevailing driver behavior and driving styles. This proposed approach evaluated multiple factors that were determinants in identifying specific driver behavior and driving styles. The results of this experiment that utilized Python, indicated that the driver model in question was successful in achieving a 90\% accuracy in terms of logistic regression.
\end{abstract}

This work is licensed under a Creative Commons Attribution 4.0 International License, which permits unrestricted use, distribution, and reproduction in any medium, provided the original work is properly cited. 
Keywords: Driver behavior; neural network algorithms; driver behavior classification; pattern analysis

\section{Introduction}

Deep learning has pioneered a new era of data and has been heralded as one of the forerunners of new and innovative statistical and computational models. Applied in the context of analyzing driver behavior, such a model can revolutionize the way driver behavior can be understood and various insights generated that can lead to real-life improvements within a short span of time. The very first step towards conducting such an analysis requires a thorough understanding and categorizations of a driver's individual skill levels and habitual patterns of driving. The various parameters that can be used for such a classification and analysis need to be defined first before the actual analysis can be carried out. For this, the availability and quality of the driving data is of utmost significance. To that end, on-board sensors or sensor-based smartphones have sometimes been used. A vehicle controller can be usually used to glean compliance related data and information including the settings of the steering wheel, vehicle speed, position of the brake pedal as well as sensor data related to the speed, direction and velocity of the vehicle.

Contemporary developments have led to such onboard data to be considered as accurate and reliable sources that can be effectively used in drive analysis and also for monitoring the behavior and style of the identified driver in question [1].

Every driver has a unique combination of speed, acceleration and braking habits that can be regarded as a fingerprint and can help identify the unique driving habit of an individual. Such a fingerprint or signature style can be extracted and identified via the analysis of an individual driver's characteristic behavior under driving conditions, such as the speed, the aggression and the focus or the lack of it. Such categorization of driving behaviors can help identify, monitor and reduce the possibility of rash or dangerous driving that can help improve long-term road safety protocols. The correlation between dangerous driving and road accidents has been long observed and is well-studied [2]. Several studies have also emphasized upon the connection between the acceleration mode and the consumption of fuel as well as hazardous fuel emissions. While these association are well accepted, a thorough understanding of driving patterns and styles with respect to various behaviors like the application of brakes under different traffic situations, for instance, have not been well understood due to the paucity of data [3].

\section{Driver Behavior Analysis}

The automotive and transportation industries, car insurance agencies, as well as government-controlled public transport infrastructure can all benefit from behavioral modeling that can promote positive driving behaviors, making such analysis an important research topic with far reaching implications. All these stakeholders have an interest in understanding such behaviors in order to be able to create innovative solution in order to improve overall performance. To that end, remedial technologies need to enable effective evaluation of parameters including driving styles as well as the driver's physical characteristics (such as face recognition, monitoring of physical features, etc.), and the use of in-vehicle telematics to monitor the collected navigation data [4].

The classification of driver behaviors is considered a complex undertaking because it is a multifaceted problem that juxtaposes these behaviors with non-uniform traffic conditions. Therefore, such an endeavor requires that variable traffic conditions, actual road conditions, vehicle-specific travel data as well as the driving behavior are effectively grouped. This has in turn necessitated the development of fuzzy sets that can enable recommendations and rules for promoting positive driving behavior specific to the driver and the drive conditions, and therefore enabling remedial operations and tactics [5]. 
Fig. 1 represents the first module of the proposed design which encapsulates the background data of the driver. Such data includes the driver's gender, age, experience, education level, work status, income level, average annual mileage, the number of drivers and violations of traffic regulations, the previous year's accident rate, etc. Once collected, a statistical learning model is trained with this data to establish a connection between the background data and accident probabilities. The model is then be used to predict the probability of an individual driver causing an accident. Grading rules are applied to background data to provide a specific driver score [6].

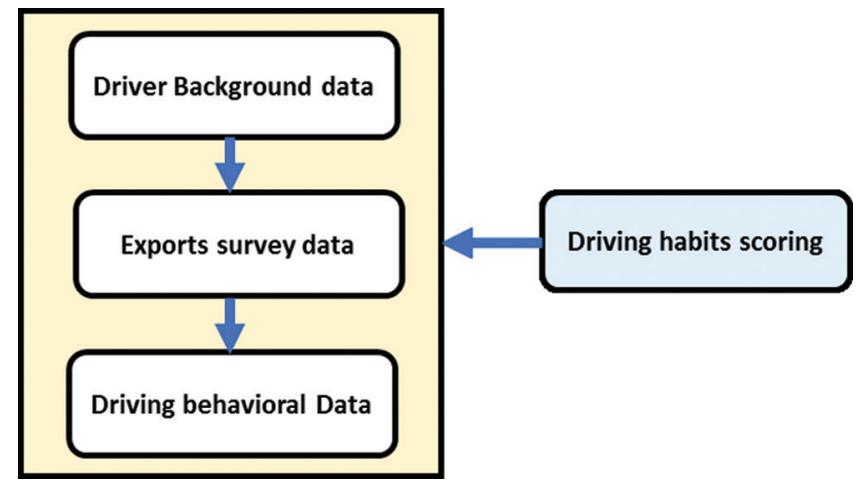

Figure 1: Driving monitoring system

In order to determine the driver's effectiveness and driving style, several factors must be evaluated and taken into account, like ecological features, road and vehicle conditions, event classification and identification, as well as natural and functional conditions. Building an accurate and thorough model that can effectively incorporate all the above mentioned aspects is a fairly complex, even unmanageable undertaking. In the past decade, a variety of commercial and research systems have been proposed with respect to driving behavior analysis in order to evaluate driving performance and to assist the driver as well as to monitor various drive systems [7].

\section{Related Work}

Taxi services propose numerous benefits and utilities to our daily lives. However, in numerous towns, the supply of taxi services fail to meet the driving demand, especially during peak hours. In this scenario, the dominant player's ambition is to expand their territory and increase mobile payment to protect its dominance. For this, one of the most important factors is to improve the company's mobile Internet for those who wish to book services online. A related example is the competition between Tencent and Alibaba becoming more intense, since their aim was to win over as many people as possible, who would pay money to facilitate taxi bookings using mobile payments [8].

Another example is the design of a dynamic surface adaptive fuzzy controller and a three-phase APF (Active Power Filter). Adaptation support methods combined with fuzzy strategies have been proposed to address the non-linearity of APF, and fuzzy controllers are the main cause for eliminating non-linearity. It is noteworthy that by using the dynamic surface method, the system may decrease the number of fuzzy input variables in modeling, design parameters and cause the controller to be made without complicated calculations [9].

Traditional biometric solutions are either few, inaccurate, slow-moving, or too expensive to put into practical use; these solutions come at the expense of precision, privacy and protection. Similarly, scalability and cost-effectiveness are inversely related. In such a system, a driver identification method may be used to solve these problems. It is usually deployed in car navigation systems to understand 
people via a global positioning system (GPS) that uses data driving mode. The current market can also use a general-purpose handheld device [10]. Halfway during the trial in each scene, the contributors were asked to accomplish the task or failed to overcome secondary distractions. In some cases, cluster analysis is used to determine a response mode drive. For driving on the highway, they increase (1) fixed variables and the steady-state performance of the eye, and (2) is fixed and constant changes, the average speed increase. Cross drives, which increases the overall speed reduction (mean and variance), then decreased at a constant speed (3) and increasing the speed, adding a fixed change [11].

To drive, a simulator is used for research, an effective tool for developing a car or driver training, which is why it is necessary to cause a similar driving behavior to suit the real car. For example, as a specific task, two minimum steering driver models of this type have been developed and tested in real data sets, a model close to the limit, low friction, and showing a clearer preference than the other. When driven by a closed-loop simulation apparatus, till the vehicle positioning data is reliable, the model parameters' current implementation is commonly used to prove a simple method of adjusting the open-loop undisturbed driver's steering data to estimate [12] accurately. Integrated signal T- intersection clustering and classification system predicts the driver's personalized, seamless control. PRM (Polynomial regression mixture) clusters and Akaike information criterion are applied to the track's driving behavior to drive indepth study. Then various categories are evaluated with respect to low vehicle conditions linked to highlevel driving behavior. CART classification Bayesian offered optimization and with excellent accuracy [13] (see Tab. 1).

First, the design and establishment of a driver behavior data acquisition system is undertaken. A different type of vehicle with a driver input data is collected under a typical operating condition signal. Drivers using PSO (Particle Swarm Optimization) algorithm is optimized to drive and use neural network behavior perception factor analysis and FCM "Fuzzy C- Means" clustering algorithm and backpropagation "BP" classification. Post that, primary caution, preparation and control system of personalized lane changes [14].

Table 1: Techniques and its limitations on driving pattern evolution

\begin{tabular}{|c|c|c|}
\hline Technique & Advantages & Disadvantages \\
\hline $\begin{array}{l}\text { Expectation maximum } \\
\text { algorithm [15] }\end{array}$ & $\begin{array}{l}\text { It proposes an expressively } \\
\text { smaller time outline }\end{array}$ & $\begin{array}{l}\text { The gaze behavior is influenced by age } \\
\text { variances, gender and driving } \\
\text { experimentations. }\end{array}$ \\
\hline $\begin{array}{l}\text { Optimization for Ranking } \\
\text { Task [16] }\end{array}$ & $\begin{array}{l}\text { Taxi driving behavior to } \\
\text { improve the predictability }\end{array}$ & $\begin{array}{l}\text { Isn't an explanation of taxi driving pattern } \\
\text { evolution }\end{array}$ \\
\hline K-means cluster analysis [17] & $\begin{array}{l}\text { Speed measurement is } \\
\text { available }\end{array}$ & $\begin{array}{l}\text { It does not dispense consistently in all } \\
\text { speed assortments }\end{array}$ \\
\hline $\begin{array}{l}\text { Hierarchical Dirichlet process } \\
\text {-hidden semi-Markov model } \\
{[18]}\end{array}$ & $\begin{array}{l}\text { It can capture static driving } \\
\text { characteristics }\end{array}$ & $\begin{array}{l}\text { The test data set is difficult to obtain } \\
\text { unknown numbers }\end{array}$ \\
\hline $\begin{array}{l}\text { Principal component analysis } \\
\text { [19] }\end{array}$ & $\begin{array}{l}\text { Classification of the driving } \\
\text { behavior and the warning to } \\
\text { the driver }\end{array}$ & $\begin{array}{l}\text { The classification evaluates only based on } \\
\text { speed }\end{array}$ \\
\hline $\begin{array}{l}\text { Hybrid Convention Neural } \\
\text { Network framework (HCF- } \\
\text { CNN) [20] }\end{array}$ & $\begin{array}{l}\text { The extracted features to } \\
\text { obtain comprehensive } \\
\text { information }\end{array}$ & This HCF-CNN not trained sufficiently \\
\hline $\begin{array}{l}\text { Automatic blink detection } \\
\text { algorithm [21] }\end{array}$ & $\begin{array}{l}\text { To reduce the dimensionality } \\
\text { of the dataset }\end{array}$ & $\begin{array}{l}\text { The warnings are often inaccurate and } \\
\text { unreliable }\end{array}$ \\
\hline
\end{tabular}


ANN (Artificial Neural Network) was used to search the composite connections between the generated road situation and the overall graphical representation's of the driving behavior, improving the deep learning model. The model Denoising Stacked Autoencoder (DSAE) laminated unsupervised can provide RGB colors for the output layer. The dataset followed for kinematics measures and is an onboard GPS device utilized for investigational operations come from Graphical results display the capability of methodologies to detect real, simple driving behavior patterns and the complexity of the road situation, environment and few actions faced on the itinerary [22].

Due to the increased number of cars and roads, there is continuing increase in the number of traffic accidents and additionally causes dangerous driver behavior. Few prevailing methodologies trust the driving behavior recognition method for joint point estimates. Still, the key point estimate cannot fully explain the human body. On the other hand, the proposed risk behavior people-oriented analysis of driving behavior recognition method can quickly and accurately identify the driver [23].

This problem targets driving behavior assessment based on extensive data network work vehicle control system to select quantitative analysis. After that, the three main indicators for a detailed description: acceleration and deceleration behavior index, an indicator of stability indicators speeding behavior and actions. Using method clustering GA-FCM "Genetic Algorithm, Fuzzy C-Means clustering", 40-level drivers of dangerous goods transport are classified according to their operating parameters. The outcomes demonstrate that most drivers at high risk of poor driving behavior and driving stability have differential levels [24].

Assessing driving behavior similarity plays an important role in driving style classification and analyzes the important role, which benefits from the design support system driven people-centered. Hidden Markov Model (HMM) is a primitive drive for automatically extracted from the observed sequence, without using the existing knowledge integrated hierarchical Dirichlet process (HDP) Bayesian non-parametric method. A relative entropy grid-based allows us to quantify the probability level of similarity between these primitives [25].

Human Posture restoration and Behavioral Analytics (HuPBA) is a computer vision, pattern analysis and challenging machine learning themes. The application includes public safety, sports, commercial, games, human-computer interaction, assistive technology, rehabilitation, recognition of handwriting and driving assistance technology. In essence, HuPBA properties need to deal with a change in appearance due to the joint body garments, such as artifacts, obstacles, and lighting background noise changes specific to the scene [26].

Machine learning algorithms, most important in revealing latest driver monitoring technology, demonstrate a close examination of basic strengths and weaknesses. Driver drowsiness and distractions: The study also falls into two categories: Driver drowsiness studies were then developed based on either visual features or invisible functions subdivided into two major subgroups [27].

For the turning lane to keep or develop advanced driver assistance systems (ADAS), an important design goal is to share driver's steering control properly. Therefore, the driver's steering behavior should be properly characterized by the designing high-performance ADAS controller. To characterize behavior of the driver's steering that system, two well-known visual-driven model, and conducted a sequence of ground trials/tests to determine model parameters and verify in real-world situations, this model [28].

For lane-keeping and growth of advanced driver assistance systems, a substantial designed goal of steering control with accurate drive share is must. Therefore, the driver's steering behavior should be high enough to characterize the performance of the controller design. In this system, the use of two wellknown video driver models to determine the model parameters and verify in the real-world field this model to characterize the driver's steering behavior [28] did a series of tests (see Tab. 2). 
Table 2: Review of existing classification methods

\begin{tabular}{|c|c|c|}
\hline Technique & Advantages & Disadvantages \\
\hline $\begin{array}{l}\text { machine-learning } \\
\text { algorithm [29] }\end{array}$ & $\begin{array}{l}\text { Provides automatic verification of } \\
\text { drivers' behavior }\end{array}$ & $\begin{array}{l}\text { Maximum times, drivers do not reduce the } \\
\text { speed }\end{array}$ \\
\hline $\begin{array}{l}\text { FCW (forward- } \\
\text { collision warning) } \\
\text { algorithm [30] }\end{array}$ & $\begin{array}{l}\text { It may regulate its cautioning threshold } \\
\text { according to the driver's behavior } \\
\text { changes. }\end{array}$ & It does not evaluate the risk perception \\
\hline $\begin{array}{l}\text { k-NN classifier } \\
{[31]}\end{array}$ & $\begin{array}{l}\text { Analyze the movement of neighboring } \\
\text { vehicles while shifting the driver's sight }\end{array}$ & $\begin{array}{l}\text { Use a longer interval without analyzing } \\
\text { gaze }\end{array}$ \\
\hline $\begin{array}{l}\text { Advanced driver } \\
\text { assistance systems } \\
\text { (ADASs) [32] }\end{array}$ & $\begin{array}{l}\text { It defines the "braking" behavior as the } \\
\text { driver's body movement }\end{array}$ & $\begin{array}{l}\text { It is difficult to identify specific drivers } \\
\text { and predict their behavior braking }\end{array}$ \\
\hline $\begin{array}{l}\text { Convolutional } \\
\text { neural network } \\
(\mathrm{CNN})[33]\end{array}$ & $\begin{array}{l}\text { Founded on the analysis of time and } \\
\text { space characteristics of the -driving state }\end{array}$ & $\begin{array}{l}\text { Adjacent levels of attention rating are not } \\
\text { clear }\end{array}$ \\
\hline $\begin{array}{l}\text { Neural network } \\
\text { algorithm [5] }\end{array}$ & $\begin{array}{l}\text { To be more observant and concurrently } \\
\text { conscious of numerous vehicles } \\
\text { positions in order to react swiftly }\end{array}$ & $\begin{array}{l}\text { The problem of sensing abnormal driver } \\
\text { behavior with the support of face netting } \\
\text { and analyzation is tough }\end{array}$ \\
\hline $\begin{array}{l}\text { Feedforward } \\
\text { neural network } \\
\text { (FFNN) [6] }\end{array}$ & $\begin{array}{l}\text { Identification task to identify evaluations } \\
\text { with different characteristics }\end{array}$ & $\begin{array}{l}\text { It is difficult to collect more real-world } \\
\text { data sets and identify deeper driver } \\
\text { behavior. }\end{array}$ \\
\hline
\end{tabular}

Detection method recommended for the driver and mobile applications are based on behavior analysis, monitoring, analysis, and personal smartphone accident prevention dangerous driving behavior. Built-in sensors (gyroscope, accelerometer, GPS, and microphone) and smartphone cameras are utilized to monitor drive activity. The method comprises the development of hazardous conditions, hazardous conditions detected, and the reference model [34].

Driver attention monitor system status and driver behavior analysis is undertaken to determine whether the driver is sympathetic. Use a front camera mounted on the windshield systems; smartphones can be used in several functions by monitoring the driver's attention. It comes from the state and is based on driver behavior, such as head posture and eye contact, eyes closed, yawning, mobile phones and other fusion using spatial and temporal characteristics of the driver's attention. Data from a metropolitan city with 30 drivers, evaluating driving data from world control, static vehicle settings [35].

First categorized, human driver's negligent driving behavior (HIDB) discussion is divided into two groupings: DD "driver distraction", DF "driven fatigue" or DF "drowsiness". After that, we deliberate the above reasons and the impact of so-called ADB "aggressive driving behavior" on other people's dangerous driving behavior. An aggressive driving style is dangerous when it leads to a large category of serious accidents. Abnormal driving behavior in individuals viz "DD", "DFD", and "ADB" impacted by numerous features which include age of driver, experience of driver, gender or insufficient ailment experience [36].

A variety of factors, fatigue, distraction, drunk, and reckless, is the most common type, resulting in unexpected driving behavior anomalies. Due to advances in technology, modern smartphones the ability to analyze driving behavior. It can only detect abnormal sleepiness and driving behavior, and the use of smartphones is not a 
comprehensive review. In this article, we discuss different approaches proposed by different authors. This method includes sensing, detection algorithms, and their respective accuracy limitations and [37].

The distracted [38] driving behavior of high frequency is considered to be a high potential risk. Turn right driver distracted driving behavior may greatly upsurge the danger of conflict. Considering complexity of the program, need is there to turn right at the intersection by the movement. Numerous aspects have been examined comprising impact of geometry of road, ecological features and driver to turn right on the traffic conditions of distracted driving activity occurring. Logistic model and RF "Random Forest" implemented for regression analysis. It was noted that lane occupancy and traffic control, two models were ominously associated to distracted driving behavior. Probability odds ratio shows an analysis of the right-turn dedicated lane, and increase production in the logo design can reduce the intersection distracted driving behavior [39].

To collected 14 data on subjective risk assessments by 14 young drivers and risk events and experienced drivers during the driving behavior of older drivers. Expert drivers classify the degree of conflict and subjective emotions in combination with the threat level of the driving sight and use fuzzy signal detection to estimate the driver's risk sensitivity " $d$ " and judgment threshold " $\beta$ ". Judgement threshold, which has largest variance between cluster analysis and driver, is classified into four forms in accordance to the threshold value. In conclusion, the driver classification identification model is founded on Fisher identification analysis [40].

In contrast, popular asymmetric characteristic provides vibrational characteristics for the general class of accurate legally unstable next-linear vehicles that are being considered to explain the recent mathematical framework of micro traffic. It is flowing with increasing interest in quantification. This method provides an angle to understand flow vibrations based on a mathematically asymmetric method, but still, it is unclear that in what way asymmetry happens [41].

\section{Materials and Methodology}

Analysis based on variable accuracy periods is based on a pre-training model that can recognize driving behavior. Though pre-trained models too have significant losses, the same cannot recognize distracted-led images without this particular dataset. This research is focused on driver behavior analysis. Initially, the collective driver dataset is pre-processed with several factors that are considered to have attributes. These prompting influences consider non-redundant data with uncovered data-filled or non-filled in a raw dataset and to verify the presence and originality of the values.

The ML algorithms were implemented in python language. The dataset was distributed into 80:20 ratios, demonstrating the training and test sets, correspondingly. Each ML algorithm's working principles are defined in the subsequent sections.

\subsection{Random Forest}

The Random forest classifier generates numerous decision trees from randomly selection subsets of the training dataset as presented in Fig. 2. Afterwards, it goes for the collection of the votes from various decision trees to elect test object's final class [42,43]. A random forest classification was projected in [44] with reduced number of trees. Fig. 2.

\subsection{Support Vector Machine (SVM)}

A SVM [45] is ML algorithm which is performed for both regression and classification tasks but is majorly utilized in classification. This classifier [46] has been used of late in numerous applications due to its excellent classifying capability and presentation excellence, distributing the data linearly into two 
separate classes (i.e., hyperplanes), with maximum distance between the two classes as Fig. 3 demonstrated below.

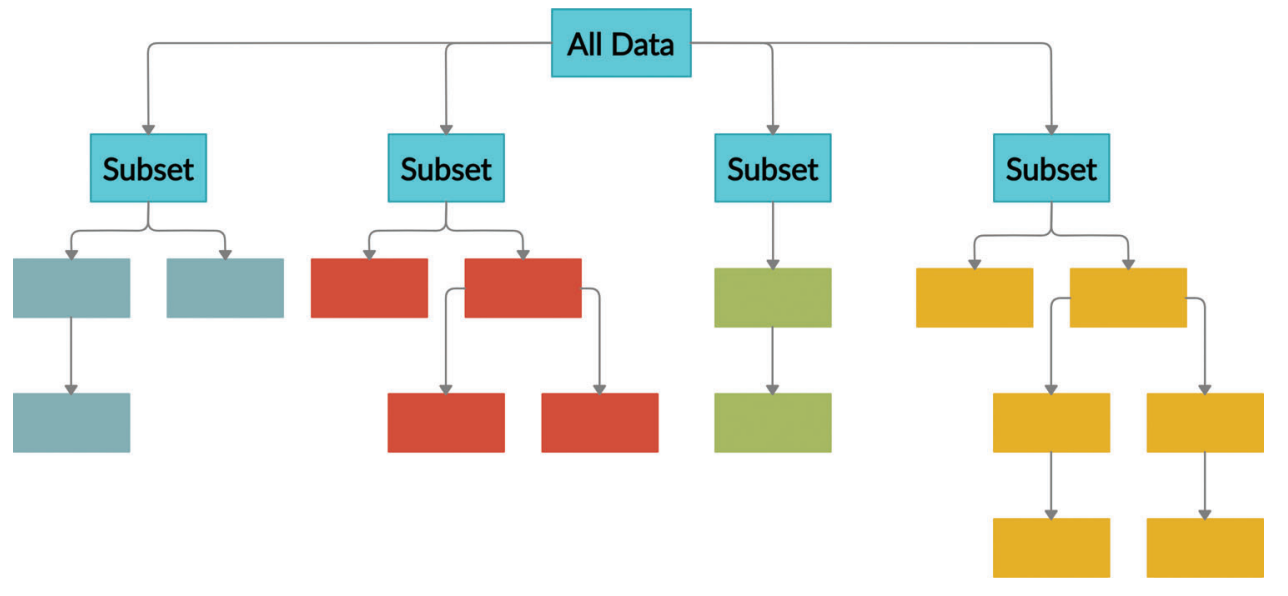

Figure 2: Random forest

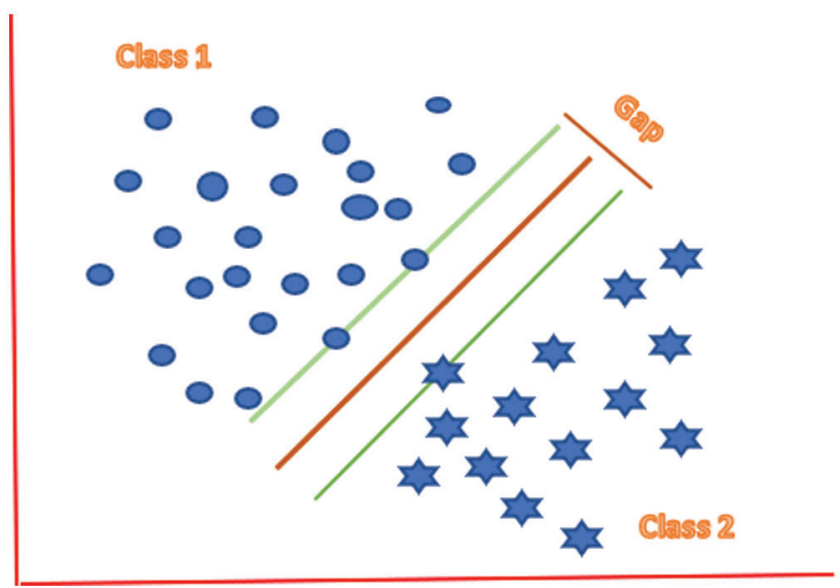

Figure 3: Support vector machine

\subsection{Logistic Regression}

Logistic regression analysis is utilized to observe the connotation of (categorical or continuous) independent variable(s) with one dichotomous dependent variable. It is in divergence to linear regression analysis where the dependent variable is a continuous variable. Logistic regression [47] is a statistical classification model which counts the association between a categorical-dependent variable (containing two categories only) and one or more independent variables, that are generally (not certainly) continuous, by utilizing probability scores as the predicted values of dependent variable. Logistic regression does not take up a linear relationship between dependent and independent variables. The independent variables require neither to be generally distributed nor linearly related or nor of equal variance within each group. 


\subsection{Gaussian Naive Bayes}

Naïve Bayes This classifier superintends ML algorithms utilizing Bayes theorem and acts on foundation whose features are analytically independent. This theorem relies on naïve assumption, where input factors are independent of each other [48-51]. Naive bayes formula is given below:

$p(H \mid D)=\frac{p(H) p(D \mid H)}{p(D)}$

Here,

$p(H \mid D)=$ It is posterior.

$p(H)=\mathrm{It}$ is the prior means what is believed before seeing the evidence.

$p(D \mid H)=$ It is the likelihood of seeing that evidence if your hypothesis is correct

$p(D)=$ It is the normalizing of that evidence under any circumstances

\section{Results and Discussions}

The application of all four approaches - i.e., RFT, Gaussian Naïve Bayes, SVM and Logistic Regression -depicted in Tab. 3. Eqs. (2)-(6) below have been used to compute accuracy and precision, recall, error rates and specificity in every confusion matrix.

Table 3: Values of different measures for different algorithms

\begin{tabular}{llllll}
\hline Methods/number of records & Precision & Sensitivity/Recall & Specificity & Accuracy & F1-score \\
\hline Logistic Regression & 0.8947 & 0.6296 & 0.9411 & 0.9016 & 0.7391 \\
SVM & 0.70 & 0.5185 & 0.8235 & 0.6885 & 0.5957 \\
Gaussian Naïve Bayes & 0.8636 & 0.7037 & 0.9117 & 0.8524 & 0.7755 \\
Random Forest Classifier & 0.7916 & 0.7037 & 0.8529 & 0.7868 & 0.7450 \\
\hline
\end{tabular}

Accuracy $=\frac{\text { Sum of diagnols }(T P)}{\text { Total number of instances }}$

Precision $=\frac{T P}{T P+F P}$

Recall $=$ Senstivity $=\frac{T P}{T P+F N}$

Specificity $=\frac{T N}{T N+F P}$

$F 1$ Score $=\frac{2 \times(\text { Precision } \times \text { Recall })}{\text { Precision }+ \text { Recall }}$

In which,

TP "True positive" stands for Diagonals of matrix

FN "False negative" stands for Sum of the consistent row for class 
FP "False positive" stands for Sum of the corresponding column for class

TN "True negative" stand for Sum of the whole row and column

Tab. 3 demonstrates the accuracy, precision, recall, error rate, specificity, sensitivity and fl score attained by the various algorithms. As conclusion of Tab. 3, the uppermost accuracy for all was achieved by logistic regression. In this type of cases, accuracy only is not an adequate degree, and the fl score counts as a significant measure for decisive selection of the best model. The $\mathrm{fl}$ score is a harmonic mean of precision and recall, having upper value, when both precision and recall are higher. Therefore, the best model in cases of classification imbalance is the one which has higher fl score even if with lower accuracy. The fl score of Random Forest was the highest for Gaussian Naïve Bayes whereas it is low for SVM. The specificity of all the algorithms was found to be around $82 \%-90 \%$ or above. Moreover, this is an important parameter. All of the algorithms applied in this study formed highly accurate outcomes.

The Tab. 3 above provides the comparison of precision, sensitivity, specificity, accuracy and F1-score. The proposed approach and logistic regression has produced a higher accuracy than any other methods. In the proposed prediction of the driver's behavioral pattern, the best pattern prediction from the driver's activities is made in order to provide the best recommendation for improving driving behaviors.

\section{Conclusion}

Driving assistance applications are increasing and driver behavior has currently become the focus of extensive research. As one of the main reasons for pushing accelerators leading to road safety issues, as research is being conducted to solve human behavior quirks and resulting safety issues. Proposing solutions through driving behavior analysis for solving road safety issues and other business problems, driver behavior represents different driving behavior modes of the same drive system, each with a different driving style. An important factor in driving monitoring systems is that, in order to detect driver behavior, we need to find an automatic collection of data and algorithms and model applications that generate classification methods that describe driving performance configuration.

The proposed technology includes the most current trends for analyzing real-world scenarios as well as classifications of driver behavior. Based on the system, various strategies can be employed to determine the target driver's behavior. The present driving behavior and driving style analysis system has been based on extensive research, and by utilizing neural network algorithms. The driver model was observed to have attained a $90 \%$ accuracy for logistic regression, according to the test findings using Python. Collecting massive volumes of driving data from various cars and drivers will be of tremendous interest in the future.

Funding Statement: The authors received no specific funding for this study.

Conflicts of Interest: The authors declare that they have no conflicts of interest to report regarding the present study.

\section{References}

[1] S. Ullah and D. H. Kim, "Lightweight driver behavior identification model with sparse learning on in-vehicle canbus sensor data," Sensors, vol. 20, no. 18, pp. 5030, 2020.

[2] J. Zhang, Z. Wu, F. Li, C. Xie, T. Ren et al., "A deep learning framework for driving behavior identification on invehicle CAN-BUS sensor data," Sensors, vol. 19, no. 6, pp. 1356, 2019.

[3] X. Zhu, X. Hu and Y. C. Chiu, "Design of driving behavior pattern measurements using smartphone global positioning system data," International Journal of Transportation Science and Technology, vol. 2, no. 4, pp. 269-288, 2013. 
[4] T. K. Chan, C. S. Chin, H. Chen and X. Zhong, "A comprehensive review of driver behavior analysis utilizing smartphones," IEEE Transactions on Intelligent Transportation Systems, vol. 21, no. 10, pp. 4444-4475, 2020.

[5] B. Shi, L. Xu, J. Hu, Y. Tang, H. Jiang et al., "Evaluating driving styles by normalizing driving behavior based on personalized driver modeling," IEEE Transactions on Systems, Man, and Cybernetics: Systems, vol. 45, no. 12, pp. 1502-1508, 2015.

[6] M. Paul, G. Sanyal, D. Samanta, G. N. Nguyen and D. N. Le, "Admission control algorithm based on the effective bandwidth in vehicle-to-infrastructure communication," IET Communications, vol. 12, no. 6, pp. 704-711, 2018.

[7] S. K. Alluhaibi, M. S. N. Al-Din and A. Moyaid, "Driver behavior detection techniques: A survey," International Journal of Applied Engineering Research, vol. 13, no. 11, pp. 8856-8861, 2018.

[8] B. Leng, H. Du, J. Wang, L. Li and Z. Xiong, "Analysis of taxi drivers' behaviors within a battle between two taxi apps," IEEE Transactions on Intelligent Transportation Systems, vol. 17, no. 1, pp. 296-300, 2016.

[9] C. Ou and F. Karray, "Enhancing driver distraction recognition using generative adversarial networks," IEEE Transactions on Intelligent Vehicles, vol. 5, no. 3, pp. 385-396, 2020.

[10] M. A. Rahim, L. Zhu, X. Li, J. Liu, Z. Zhang et al., "Zero-to-stable driver identification: A non-intrusive and scalable driver identification scheme," IEEE Transactions on Vehicular Technology, vol. 69, no. 1, pp. 163$171,2020$.

[11] Y. Liao, G. Li, S. E. Li, B. Cheng and P. Green, "Understanding driver response patterns to mental workload increase in typical driving scenarios," IEEE Access, vol. 6, pp. 35890-35900, 2018.

[12] G. Markkula, R. Romano, A. H. Jamson, L. Pariota, A. Bean et al., "Using driver control models to understand and evaluate behavioral validity of driving simulators," IEEE Transactions on Human-Machine Systems, vol. 48, no. 6, pp. 592-603, 2018.

[13] D. Yi, J. Su, C. Liu and W. H. Chen, "Trajectory clustering aided personalized driver intention prediction for intelligent vehicles," IEEE Transactions on Industrial Informatics, vol. 15, no. 6, pp. 3693-3702, 2019.

[14] B. Zhu, S. Yan, J. Zhao and W. Deng, "Personalized lane-change assistance system with driver behavior identification," IEEE Transactions on Vehicular Technology, vol. 67, no. 11, pp. 10293-10306, 2018.

[15] R. Zheng, K. Nakano, H. Ishiko, K. Hagita, M. Kihira et al., "Eye-gaze tracking analysis of driver behavior while interacting with navigation systems in an urban area," IEEE Transactions on Human-Machine Systems, vol. 46, no. 4, pp. 546-556, 2016.

[16] T. Xu, H. Zhu, H. Xiong, H. Zhong and E. Chen, "Exploring the social learning of taxi drivers in latent vehicle-tovehicle networks," IEEE Transactions on Mobile Computing, vol. 19, no. 8, pp. 1804-1817, 2019.

[17] D. Wang, X. Pei, L. Li and D. Yao, "Risky driver recognition based on vehicle speed time series," IEEE Transactions on Human-Machine Systems, vol. 48, no. 1, pp. 63-71, 2018.

[18] W. Wang, J. Xi and D. Zhao, "Driving style analysis using primitive driving patterns with Bayesian nonparametric approaches," IEEE Transactions on Intelligent Transportation Systems, vol. 20, no. 8, pp. 2986-2998, 2019.

[19] J. M. Escaño, M. A. Ridao-Olivar, C. Ierardi, A. J. Sánchez and K. Rouzbehi, "Driver behavior soft-sensor based on neurofuzzy systems and weighted projection on principal components," IEEE Sensors Journal, vol. 20, no. 19, pp. 11454-11462, 2020.

[20] C. Huang, X. Wang, J. Cao, S. Wang and Y. Zhang, "HCF: A hybrid CNN framework for behavior detection of distracted drivers," IEEE Access, vol. 8, pp. 109335-109349, 2020.

[21] H. Mårtensson, O. Keelan and C. Ahlström, "Driver sleepiness classification based on physiological data and driving performance from real road driving," IEEE Transactions on Intelligent Transportation Systems, vol. 20, no. 2, pp. 421-430, 2019.

[22] A. Bichicchi, R. Belaroussi, A. Simone, V. Vignali, C. Lantieri et al., "Analysis of road-user interaction by extraction of driver behavior features using deep learning," IEEE Access, vol. 8, pp. 19638-19645, 2020.

[23] C. Zhang, Y. Lu, M. Feng and M. Wu, "Trucker behavior security surveillance based on human parsing," IEEE Access, vol. 7, pp. 97526-97535, 2019.

[24] X. Wang and H. Wang, "Driving behavior clustering for hazardous material transportation based on genetic fuzzy c-means algorithm," IEEE Access, vol. 8, pp. 11289-11296, 2020. 
[25] W. Wang, W. Han, X. Na, J. Gong and J. Xi, "A probabilistic approach to measuring driving behavior similarity with driving primitives," IEEE Transactions on Intelligent Vehicles, vol. 5, no. 1, pp. 127-138, 2020.

[26] S. D. Camera, "Special issue on multimodal human pose recovery and behavior analysis," IEEE Transactions on Pattern Analysis and Machine Intelligence, vol. 38, no. 8, pp. 1489-1491, 2016.

[27] S. Kaplan, M. A. Guvensan, A. G. Yavuz and Y. Karalurt, "Driver behavior analysis for safe driving: A survey," IEEE Transactions on Intelligent Transportation Systems, vol. 16, no. 6, pp. 3017-3032, 2015.

[28] C. You, J. Lu and P. Tsiotras, "Nonlinear driver parameter estimation and driver steering behavior analysis for ADAS using field test data," IEEE Transactions on Human-Machine Systems, vol. 47, no. 5, pp. 686-699, 2017.

[29] A. Bouhoute, R. Oucheikh, K. Boubouh and I. Berrada, "Advanced driving behavior analytics for an improved safety assessment and driver fingerprinting," IEEE Transactions on Intelligent Transportation Systems, vol. 20, no. 6, pp. 2171-2184, 2019.

[30] J. Wang, C. Yu, S. E. Li and L. Wang, "A forward collision warning algorithm with adaptation to driver behaviors," IEEE Transactions on Intelligent Transportation Systems, vol. 17, no. 4, pp. 1157-1167, 2016.

[31] T. Hirayama, K. Mase, C. Miyajima and K. Takeda, "Classification of driver's neutral and cognitive distraction states based on peripheral vehicle behavior in driver's gaze transition," IEEE Transactions on Intelligent Vehicles, vol. 1, no. 2, pp. 148-157, 2016.

[32] B. Wu, Y. Zhu, S. Nishimura and Q. Jin, "Analyzing the effects of driving experience on prebraking behaviors based on data collected by motion capture devices," IEEE Access, vol. 8, pp. 197337-197351, 2020.

[33] I. Dua, A. U. Nambi, C. V. Jawahar and V. N. Padmanabhan, "Evaluation and visualization of driver inattention rating from facial features," IEEE Transactions on Biometrics, Behavior, and Identity Science, vol. 2, no. 2, pp. 98-108, 2019.

[34] A. Kashevnik, I. Lashkov and A. Gurtov, "Methodology and mobile application for driver behavior analysis and accident prevention," IEEE Transactions on Intelligent Transportation Systems, vol. 21, no. 6, pp. 2427-2436, 2020.

[35] S. K. Prasad, J. Rachna, O. I. Khalaf and D. N. Le, "Map matching algorithm: Real time location tracking for smart security application," Telecommunications and Radio Engineering, vol. 79, no. 13, pp. 1189-1203, 2020.

[36] M. H. Alkinani, W. Z. Khan and Q. Arshad, "Detecting human driver inattentive and aggressive driving behavior using deep learning: Recent advances, requirements and open challenges," IEEE Access, vol. 8, pp. 105008105030, 2020.

[37] T. K. Chan, C. S. Chin, H. Chen and X. Zhong, "A comprehensive review of driver behavior analysis utilizing smartphones," IEEE Transactions on Intelligent Transportation Systems, vol. 21, no. 10, pp. 4444-4475, 2020.

[38] K. Srinivasan, L. Garg, D. Datta, A. A. Alaboudi, N. Z. Jhanjhi et al., "Performance comparison of deep cnn models for detecting driver's distraction," Computers, Materials \& Continua, vol. 68, no. 3, pp. 4109-4124, 2021.

[39] B. Lv, R. Yue and Y. Zhang, "The Influence of different factors on right-turn distracted driving behavior at intersections using naturalistic driving study data," IEEE Access, vol. 7, pp. 137241-137250, 2019.

[40] D. Ni, F. Guo, Y. Zhou and C. Shi, "Determination of risk perception of drivers using fuzzy-clustering analysis for road safety," IEEE Access, vol. 8, pp. 125501-125512, 2020.

[41] Q. Wan, G. Peng, Z. Li, F. Inomata, Y. Zheng et al., "Using asymmetric theory to identify heterogeneous drivers' behavior characteristics through traffic oscillation," IEEE Access, vol. 7, pp. 106284-106294, 2019.

[42] V. F. Rodriguez-Galiano, B. Ghimire, J. Rogan, M. Chica-Olmo and J. P. Rigol-Sanchez, "An assessment of the effectiveness of a random forest classifier for land-cover classification," ISPRS Journal of Photogrammetry and Remote Sensing, vol. 67, no. 4, pp. 93-104, 2012.

[43] T. N. Bao, Q. T. Huynh, X. T. Nguyen, G. N. Nguyen and D. N. Le, "A novel particle swarm optimization approach to support decision-making in the multi-round of an auction by game theory," International Journal of Computational Intelligence Systems, vol. 13, no. 1, pp. 1447-1463, 2020.

[44] A. Paul, D. P. Mukherjee, P. Das, A. Gangopadhyay, A. R. Chintha et al., "Improved random forest for classification," IEEE Transactions on Image Processing, vol. 27, no. 8, pp. 4012-4024, 2018.

[45] C. Cortes and V. Vapnik, "Support-vector networks," Machine Learning, vol. 20, no. 3, pp. 273-297, 1995. 
IASC, 2022, vol.32, no.1

[46] T. Hamed, R. Dara and S. C. Kremer, "An accurate, fast embedded feature selection for SVMs," in 2014 13th International Conference on Machine Learning and Applications (ICMLA), Detroit, MI, USA, IEEE, pp. 135-140, 2014.

[47] S. Dreiseitl and L. Ohno-Machado, "Logistic regression and artificial neural network classification models: A methodology review," Journal of Biomedical Informatics, vol. 35, no. 5-6, pp. 352-359, 2002.

[48] M. Malik, R. Nandal, S. Dalal, V. Jalglan and D. Le, "Driving pattern profiling and classification using deep learning," Intelligent Automation \& Soft Computing, vol. 28, no. 3, pp. 887-906, 2021.

[49] Anatoliy Zabrovskiy, Prateek Agrawal, Roland Matha, Christian Timmerer and Radu Prodan, "ComplexCTTP: Complexity Class Based Transcoding Time Prediction for Video Sequences Using Artificial Neural Network," in 6th IEEE Conf. on Big data in Multimedia (BigMM’20), New Delhi, IEEEXplore, pp. 316-325, 2020.

[50] Prateek Agrawal, Vishu Madaan, Dimple Sethi, Naveen Kundu and Sanjay Kumar Singh, "X-HuBIS: A fuzzy rule based human behaviour identification system based on body gestures," Indian Journal of Science and Technology, vol. 9, no. 44, pp. 1-6, 2016.

[51] Charu Gupta, Prateek Agrawal, Rohan Ahuja, Kunal Vats, Chirag Pahuja et al., "Pragmatic Analysis of Classification Techniques based on Hyperparameter Tuning for Sentiment Analysis," in Int. Semantic Intelligence Conf. (ISIC'21), Delhi, pp. 453-459, 2021. 